HETEROCYCLES, Vol. 83, No. 10, 2011, pp. 2271 - 2274. @ 2011 The Japan Institute of Heterocyclic Chemistry Received, 11th July, 2011, Accepted, 11th August, 2011, Published online, 18th August, 2011 DOI: $10.3987 / C O M-11-12303$

\title{
THE [2+2] CYCLOADDITION REACTION OF ETHYNYLATED 2H-CYCLOHEPTA[b]FURAN-2-ONES WITH 2,3-DICHLORO-5,6- DICYANO-1,4-BENZOQUINONE
}

\section{Taku Shoji, ${ }^{\mathrm{a} *}$ Junya Higashi, ${ }^{\mathrm{b}}$ Shunji Ito, ${ }^{\mathrm{c}}$ Masafumi Yasunami, ${ }^{\mathrm{d}}$ and Noboru Morita ${ }^{\mathrm{b}}$ *}

a Department of Chemistry, Faculty of Science, Shinshu University, Matsumoto, 390-8621, Japan. E-mail: tshoji@shinshu-u.ac.jp

b Department of Chemistry, Graduate School of Science, Tohoku University, Sendai 980-8578, Japan. E-mail: nmorita-kamo@hb.tp1.jp

c Graduate School of Science and Technology, Hirosaki University, Hirosaki 036-8561, Japan

d Department of Materials Chemistry and Engineering, College of Engineering, Nihon University, Koriyama 963-8642, Japan

\begin{abstract}
Ethynylated 2H-cyclohepta[b]furan-2-ones reacted with 2,3-dichloro-5,6-dicyano-1,4-benzoquinone (DDQ) in a formal [2+2] cycloaddition reaction to afford the corresponding DDQ-adducts 10-15 in good yields.
\end{abstract}

2,3-Dichloro-5,6-dicyano-1,4-benzoquinone (DDQ) is well known as an oxidizing reagent by the formal dehydrogenation reaction in organic synthesis. ${ }^{1}$ Recently, Diederich ${ }^{2}$ and Trofimov $^{3}$ have reported $[2+2]$ cycloaddition reaction of DDQ with various alkyne derivatives to give the corresponding cycloadducts. They have also reported the unusual reactivities and properties of the DQQ-adducts, such as rearrangement of the carbon skeleton, ${ }^{2,4}$ intramolecular charge-transfer absorption and third-order optical nonlinearities. ${ }^{2}$ These results show the DQQ-adducts have a potential as a new series of advanced organic materials.

$2 H$-Cyclohepta[ $b]$ furan-2-one is known as a heteroazulene, a versatile precursor for azulene derivatives. ${ }^{5}$ However, there are few derivatives for the application to the advanced materials with potentially useful electronic properties. For the extension of this chemistry, we have focused our studies on the synthesis of 
$2 \mathrm{H}$-cyclohepta[b]furan-2-one-substituted tetracyanobutadiene (TCBD) derivatives by the [2+2] cycloaddition reaction of the corresponding alkynes with tetracyanoethylene. ${ }^{6}$ The study revealed the TCBD derivatives are featured by a strong ICT absorption in their UV/Vis spectra. Arylethynyl-2H-cyclohepta[b]furan-2-ones 1-9 are also expected to show high reactivity with DDQ toward the [2+2] cycloaddition reaction to afford the novel DDQ-adducts due to the electron-donating nature of the $2 H$-cyclohepta $[b]$ furan-2-one moieties. Moreover, the DDQ-adducts with $2 H$-cyclohepta[b]furan-2-one substituents are also expected to show the third-order optical nonlinearities. Herein, we describe the synthesis of the DDQ-adducts with $2 H$-cyclohepta[ $b]$ furan-2-one substituents by the formal [2+2] cycloaddition reaction of arylethynyl-2H-cyclohepta[b]furan-2-ones 1-9 with DDQ. The arylethynyl-2H-cyclohepta[b]furan-2-ones 1-9 were prepared by Sonogashira-Hagihara reaction, according to the procedure reported by us, recently. ${ }^{5}$ To the synthesis of novel DDQ-adducts, [2+2] cycloaddition reaction of ethynyl substituted $2 H$-cyclohepta[b]furan-2-ones 1-9 with DDQ was examined according to the procedure described in the literature. ${ }^{2,3}$ General experimental procedure was shown in the section of REFERENCE AND NOTES. ${ }^{7}$ The yield of the products was summarized in Table 1.

The reaction of 1 with DDQ in refluxing ethyl acetate yielded $\mathbf{1 0}$ in $83 \%$ yield. Acetylene derivatives 2 and $\mathbf{3}$ were also readily reacted with DDQ to afford the corresponding cycloadducts $\mathbf{1 1}$ and $\mathbf{1 2}$ in $70 \%$ and $84 \%$ yields, respectively, as similar to the reaction of 1 with DDQ. In the reaction of acetylene derivatives with TCNE, the [2+2] cycloaddition with an alkyne moiety afford the cyclobutene intermediate, following to the ring-opening reaction of the cyclobutene derivative gives the thermodynamically stable TCBDs. ${ }^{5 c, 6}$ Stability of cyclobutene in DDQ-adducts might be attributable to the structural effect of fused cyclohexene ring. To investigate the effect of the substituent on the benzene ring, the reaction of $\mathbf{4 , 5}$ and 6, which possess electron-withdrawing and electron-donating aryl groups, respectively, on the acetylene terminal, were examined under similar reaction conditions. Although the cycloaddition reaction of $\mathbf{4}$ with DDQ afforded the corresponding product $\mathbf{1 3}$ in 74\% yield, reaction of $\mathbf{5}$ and $\mathbf{6}$ gave inseparable complex mixtures instead of the desired cycloaddition products. Furthermore, compounds $\mathbf{5}$ and $\mathbf{6}$ did not react with DDQ at room temperature and starting materials were completely recovered. Similar with $\mathbf{5}$ and $\mathbf{6}$, ferrocene-substituted alkyne 7 did not generate the presumed cycloaddition product by the reaction with DDQ in ethyl acetate at the refluxing temperature. These results indicate the highly electron-donating aryl group on the acetylene terminal should be prevented the desired [2+2] cycloaddition reaction of acetylene moiety with DDQ. The [2+2] cycloaddition reaction of thiophene-substituted alkyne 8 with DDQ also afforded the corresponding cycloadduct 14 in $76 \%$ yield, under the similar reaction conditions. The reaction of symmetrically substituted alkyne $\mathbf{9}$ with DDQ yielded the corresponding cycloadduct $\mathbf{1 5}$ in 59\% yield. These novel DDQ adducts 10-15 are obtained as stable crystals and can be storable in the crystalline state under ordinary conditions. 


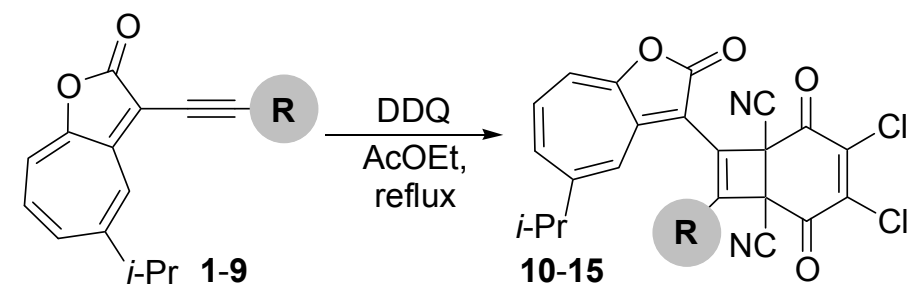

\section{Scheme 1}

Table 1. Reaction of Ethynylated 2H-cyclohepta[b]furan-2-ones 1-9 with DDQ

\begin{tabular}{llll}
\hline Substrate & $\mathrm{R}$ & Reaction time $[\mathrm{h}]$ & Product, Yield [\%] \\
\hline $\mathbf{1}$ & $\mathrm{H}$ & 12 & $\mathbf{1 0}, 83$ \\
$\mathbf{2}$ & $\mathrm{SiMe}_{3}$ & 10 & $\mathbf{1 1}, 70$ \\
$\mathbf{3}$ & phenyl & 14 & $\mathbf{1 2 , 8 4}$ \\
$\mathbf{4}$ & $p$-nitrophenyl & 18 & $\mathbf{1 3}, 74$ \\
$\mathbf{5}$ & $p$-anilino & $10 \mathrm{~min}$ & $\mathbf{-}$ \\
$\mathbf{6}$ & $p$ - $N$,N-(dimethylanimo)phenyl & $10 \mathrm{~min}$ & - \\
$\mathbf{7}$ & ferrocenyl & $10 \mathrm{~min}$ & - \\
$\mathbf{8}$ & 2-thienyl & 7 & $\mathbf{1 4 , 7 6}$ \\
$\mathbf{9}$ & 5-isopropyl-2-oxo-2H-cyclohepta[b]fur-3-yl & 1 & $\mathbf{1 5}, 59$ \\
\hline
\end{tabular}

These new compounds 10-15 were fully characterized by the spectral data. Mass spectra of 10-15 ionized by ESI showed the correct molecular ion peaks observed as $[\mathrm{M}+\mathrm{Na}]^{+}$ion peaks. The

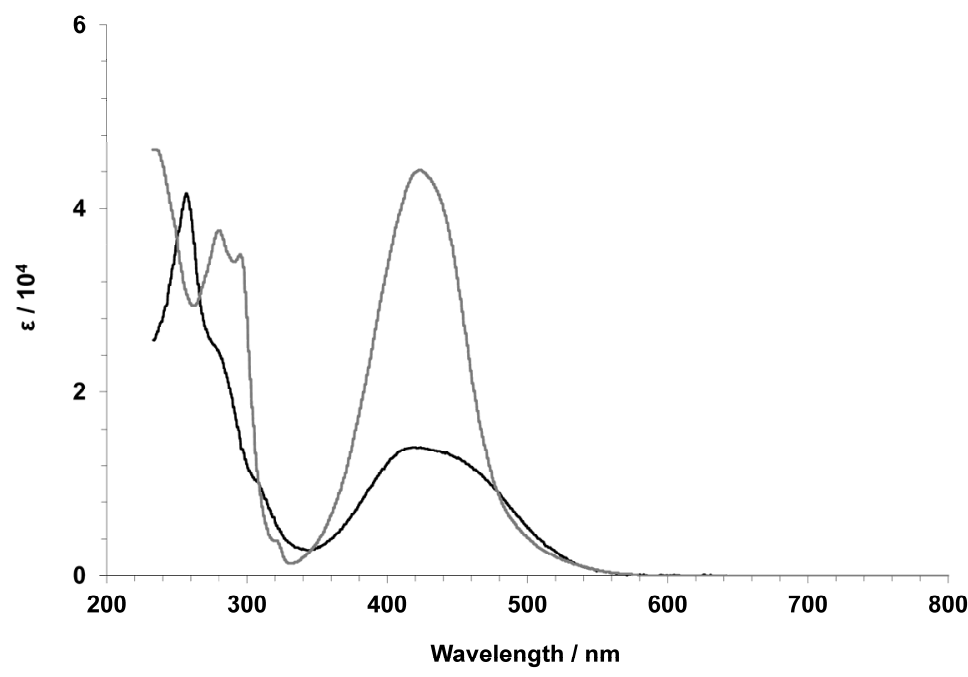

Figure 1. The UV/Vis spectra of $\mathbf{3}$ (gray line) and $\mathbf{1 2}$ (black line) in dichloromethane characteristic stretching vibration band of the $\mathrm{C} \equiv \mathrm{N}$ moieties of $\mathbf{1 0}-\mathbf{1 5}$ was observed at $v=2191-2225 \mathrm{~cm}^{-1}$ on their IR spectra, instead of the characteristic stretching band for $\mathrm{C} \equiv \mathrm{C}$ triple bond of the starting acetylene derivatives. The UV/Vis spectra of 3 and $\mathbf{1 2}$ in dichloromethane are shown in Figure 1. The absorption maxima of the acetylene derivative 3 showed a relatively strong absorption band at $426 \mathrm{~nm} \cdot 6^{6}$ On the other hand, the DDQ-adduct $12\left(\lambda_{\max }\right.$ $=418 \mathrm{~nm}$ ) showed hypsochromic shift by 
about $8 \mathrm{~nm}$, compared with that of 3 . The blue-shift of 12 might be ascribed to the less effective conjugation owing to the low planarity between $2 H$-cyclohepta $[b]$ furan-2-one and aryl moieties.

In conclusion, the novel DDQ-adducts with $2 H$-cyclohepta[ $b]$ furan-2-one substituents $\mathbf{1 0}-\mathbf{1 5}$ were synthesized by the [2+2] cycloaddition reaction of the arylethynyl- $H$-cyclohepta[b]furan-2-ones 1-9 with DDQ in good yields, although the electron-rich arene-substituted 5-7 did not afford the presumed cycloaddition products. To evaluate the value of the novel DDQ-adducts, reactivities, spectroscopic, electrochemical and theoretical aspects of these new compounds are currently progress in our laboratory.

\section{ACKNOWLEDGEMENTS}

This work was partially supported by a Grant-in-Aid for Research Activity Start-up (Grant 22850007 to T.S.) from the Ministry of Education, Culture, Sports, Science, and Technology, Japan.

\section{REFERENCES AND NOTES}

1. D. Walker and J. D. Hiebert, Chem. Rev., 1967, 67, 153.

2. S. Kato, M. T. R. Beels, P. La Porta, W. B. Schweizer, C. Boudon, J.-P. Gisselbrecht, I. Biaggio, and F. Diederich, Angew. Chem. Int. Ed., 2010, 49, 6207.

3. B. A. Trofimov, L. N. Sobenina, Z. V. Stepanova, I. A. Ushakov, L. M. Sinegovskaya, T. I. Vakul'skaya, and A. I. Mikhaleva, Synthesis, 2010, 470.

4. (a) B. A. Trofimov, L. N. Sobenina, Z. V. Stepanova, I. A. Ushakov, A. I. Mikhaleva, D. N. Tomilin, O. N. Kazheva, G. G. Alexandrov, A. N. Chekhlov, and O. A. Dyachenko, Tetrahedron Lett., 2010, 51, 5028; (b) L. N. Sobenina, Z. V. Stepanova, I. A. Ushakov, A. I. Mikhaleva, D. N. Tomilin, O. N. Kazheva, G. G. Alexandrov, O. A. Dyachenko, and B. A. Trofimov, Tetrahedron, 2011, 67, 4832.

5. (a) K. Takase and M. Yasunami, J. Syn. Org. Chem. Jpn., 1981, 39, 1172; (b) N. Morita, K. Toyota, and S. Ito, Heterocycles, 2009, 78, 1917; (c) T. Shoji, S. Ito, K. Toyota, M. Yasunami, and N. Morita, Chem. Eur. J., 2008, 14, 8398.

6. T. Shoji, J. Higashi, S. Ito, T. Okujima, M. Yasunami, and N. Morita, Chem. Eur. J., 2011, $17,5116$.

7. To a solution of 1-9 in AcOEt was added 1.1-1.6 equiv. of DDQ. The resulting mixture was refluxed for 1-18 $\mathrm{h}$ under an Ar atmosphere. The solvent was removed under reduced pressure. The residue was purified by column chromatography on silica gel to give corresponding DDQ-adducts 10-15 (The yield of the products is shown in Table 1). 